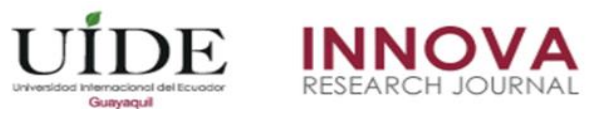

INNOVA Research Journal, ISSN 2477-9024

(Diciembre, 2017) Vol. 2, No.12 pp. 99-111

DOI: https://doi.org/10.33890/innova.v2.n12.2017.309

URL: http://revistas.uide.edu.ec/index.php/innova/index

Correo: innova@uide.edu.ec

\title{
Evaluación del proceso de obtención de biodiesel por catálisis heterogénea a partir de la grasa de pollo recuperada de los residuos generados en el proceso de cocción
}

\section{Evaluation of the process of obtaining biodiesel by heterogeneous catalysis from the chicken fat recovered from the waste generated in the cooking process}

\author{
Andrés Guayara \\ Ana Rodríguez \\ Jefferson Rubio \\ Walberto Gallegos \\ Universidad Internacional SEK Ecuador, Ecuador \\ Autor de correspondencia:walberto.gallegos@gmail.com \\ Fecha de recepción: 03 de julio de 2017 - Fecha de aceptación: 30 de noviembre de 2017
}

Resumen: Se analizaron las mejores condiciones experimentales para la obtención de biodiesel a partir de la grasa de pollo recuperada del proceso de cocción de los pollos horneados que se comercializan en una cadena de supermercados de la ciudad de Quito. La transesterificación se realizó por catálisis heterogénea comparando la efectividad del óxido de calcio y óxido de magnesio como catalizadores. La fase experimental se desarrolló con un diseño factorial 23, las variables de estudio fueron: la relación molar alcohol-aceite, el tipo de catalizador y su porcentaje en peso. Las mejores condiciones de reacción para ambos catalizadores resultaron de una relación molar alcohol-aceite 12:1 y 1\% de catalizador referido a la masa de grasa. Las máximas conversiones a biodiesel en los tratamientos con óxido de magnesio y de calcio fueron respectivamente del 22,17\% y del 90,20\%. El biocombustible obtenido bajo las mejores condiciones de operación cumplió con los requerimientos establecidos por el Instituto Ecuatoriano de Normalización (INEN) de acuerdo a la norma INEN 1489 para el Diésel No.2, el cual es utilizado en automotores a diésel.

Palabras clave: ácidos; grasos libres; catálisis heterogénea; transesterificación; triglicéridos

\begin{abstract}
The investigation was oriented to determine the best experimental conditions to produce biodiesel using as raw material chicken grease that was recuperated from the baking process used in a local supermarket chain. The transesterification reaction compared the effectiveness of calcium and magnesium oxide as catalysts. A factorial design 23 was defined for the experimental face. The factors investigated were: molar proportion between methanol and oil, type of catalyst and it's weight percentage. The best experimental conditions for both catalysts were a molar proportion methanol-oil $12: 1$ and $1 \%$ of catalyst referred to oil's mass. The maximum yields obtained for treatments with magnesium and calcium oxide were 22, $17 \%$ and 90, 20\% respectively. Regarding the biofuel's quality; it fulfilled all the requirements of standard INEN 1489 for Diesel No.2 established by the Ecuadorian Service of Normalization INEN.
\end{abstract}

Key words: transesterification; heterogeneous catalysis; saponification; triglycerides 


\section{Introducción}

Los combustibles fósiles han jugado un papel fundamental a lo largo de la historia supliendo necesidades energéticas básicas del hombre. La demanda mundial de estos recursos no renovables se ha incrementado significativamente generando una fuerte dependencia y excesivo consumo de los mismos, al punto que cada año se utiliza una cantidad de petróleo cuatro veces superior a la que se descubre (Dinis, 2012).

Se suma a esta realidad una mayor conciencia pública sobre los impactos ambientales y los potenciales peligros a la salud producto de las emisiones generadas por la quema de estos combustibles (Dinis, 2012).

Una de las soluciones más atractivas ha sido el biodiesel que proviene de fuentes renovables, tiene buenos resultados en motores a diésel y su utilización disminuye emisiones contaminantes. De acuerdo a estudios del National Biodiesel Bord, asociación que representa la industria de biodiesel en EE. UU, se determinó que su combustión emite en promedio $48 \%$ menos de monóxido de carbono, $47 \%$ menos material particulado y $67 \%$ menos hidrocarbono al ambiente que el petrodiesel (Medina et al 2013).

El proceso de síntesis más empleado para su elaboración es la transesterificación; reacción en la cual los triglicéridos de un aceite vegetal o una grasa animal reaccionan con un alcohol en presencia de un catalizador para dar lugar a la formación de esteres alquílicos (biodiesel) y glicerina como subproducto (Alba, 2011).

La transesterificación con catalizadores heterogéneos tanto ácidos como básicos se ha estudiado en los últimos años como una alternativa prometedora. La implementación de estos sistemas catalíticos permite simplificar los procesos de purificación, minimizar los efluentes contaminantes, reutilizar los catalizadores y por tanto minimizar costos de operación. Adicionalmente, es posible la conversión de una mayor gama de sustancias grasas incluyendo aquellas que muestren mayor degradación como es el caso del aceite de frituras o grasa animal residual (Becerra et al, 2008).

Según Ramírez et al (2012), existen reportes que remarcan la eficiencia de la catálisis heterogénea en comparación con la homogénea, ya que se observó que para la producción de 8000 toneladas de biodiesel fue necesario emplear 88 toneladas de hidróxido de sodio, mientras que solo se requirieron 5,7 toneladas de óxido de magnesio soportado para esta producción.

En esta investigación se planteó la obtención de biodiesel empleando transesterificación por catálisis heterogénea a partir de grasa animal. Se propuso determinar las condiciones óptimas de reacción que permitan alcanzar altos rendimientos en base a catalizadores accesibles y de bajo costo como son el óxido de calcio y óxido de magnesio. La calidad del biodiesel obtenido se evaluó mediante los parámetros establecidos en la norma INEN 1489.

Con relación a la materia prima, se optó por el aprovechamiento de un residuo como es la grasa proveniente del proceso de cocción de los pollos horneados comercializado en una de las sucursales de la cadena de supermercados ubicada en la ciudad de Quito. 
La producción del biocombustible empleando grasa de pollo residual se alinea a los principios de la producción más limpia al aprovechar un subproducto útil, lo cual conlleva a múltiples ventajas tales como: la mitigación de impactos ambientales, generación de bienestar social al contar con un ambiente más sano, incremento de la competitividad por los bajos costos de la materia prima y por tanto importantes oportunidades de emprendimiento (Galeano y Guapacha, 2011).

Las condiciones óptimas determinadas experimentalmente sirvieron para la estimación de la capacidad productiva de biodiesel con la que cuentan las sucursales ubicadas en Quito; lo cual constituye un aporte para la realización de un eventual estudio de prefactibilidad con miras a la implementación de este sistema productivo.

\section{Materiales y métodos}

\section{Diseño Experimental:}

Las variables de estudio seleccionadas para la transesterificación fueron: la relación molar metanol-aceite (RMAC), tipo de catalizador y su porcentaje en peso. Se eligieron dos niveles de prueba por cada factor (tabla 1) lo cual permitió establecer un diseño factorial 23 que comprendió la realización de ocho tratamientos combinando aleatoriamente los niveles asignados a las variables. Se realizó una repetición generando un total de 16 pruebas.

Tabla 1: Descripción de variables y sus niveles

\begin{tabular}{lcc}
\hline Variables de Estudio & Nivel & Nivel 2 \\
\hline Relación molar Alcohol: Aceite (RMAC) & $6: 1$ & $12: 1$ \\
Porcentaje en peso del Catalizador (PPC) & 1 & 2 \\
Tipo de Catalizador (TC) & $\mathrm{MgO}$ & $\mathrm{CaO}$ \\
\hline
\end{tabular}

Las variables controladas fueron, temperatura de $60 \mathrm{oC} \pm 2 \mathrm{oC}$, tiempo de reacción de 180 minutos y agitación de 1200 RPM. Se eligió como variable de respuesta al rendimiento de la reacción, el cual se calculó en base al volumen de biodiesel obtenido por tratamiento tras su respectiva purificación.

\section{Proceso Experimental:}

El proceso experimental constó de cuatro fases: muestreo, caracterización de la materia prima, pre-tratamiento de reactivos y la elaboración de biodiesel con su respectiva caracterización.

\section{Etapa de Muestreo:}


El muestreo se realizó mensualmente entre marzo y mayo de 2016. La grasa de pollo residual se obtuvo de una de las sucursales de la cadena de supermercados. La misma se genera durante la cocción de los pollos en un horno con capacidad de 34 aves por lote. La grasa se recupera en bandejas dispuestas horizontalmente en su interior y se almacena en bidones para su posterior disposición. Mensualmente se producen 90 litros de grasa.

Se tomaron seis muestras durante el período de investigación cada una de cuatro litros, las mismas eran tomadas directamente de las bandejas internas del horno al culminarse un lote de pollos horneados. El contenido era vertido en un recipiente plástico mediante el uso de un embudo y posteriormente transportado al Laboratorio de Química de la Universidad Internacional SEK para ensayos ulteriores.

\section{Caracterización de la grasa residual recuperada del proceso de cocción:}

La caracterización de la materia prima permitió determinar la idoneidad de la grasa para el proceso de transesterificación. La tabla 2 resume ensayos que fueron efectuados y la normativa seguida para la realización de cada uno.

Tabla 2: Ensayos de caracterización

\begin{tabular}{lc}
\hline \multicolumn{1}{c}{ Ensayo } & Norma \\
\hline Índice de Saponificación & INEN 40 \\
Índice de peróxido & INEN 277 \\
Índice de Acidez & INEN 38 \\
Perfil Lipídico & AOAC 996.06 \\
\hline
\end{tabular}

\section{Pre tratamiento de los reactivos:}

a) Pretratamiento de la grasa de pollo residual recuperada del proceso de cocción:

El contenido de ácidos grasos libres fue el criterio para la selección del pre tratamiento. A valores iguales o mayores al 3\% en masa se debe realizar su esterificación (Rojas et al, 2009). Considerando que el índice de acidez obtenido fue inferior $(0,1 \%)$ no fue necesaria la implementación de este tratamiento. El acondicionamiento de la materia prima se limitó sólo a filtración al vacío.

b) Activación del Catalizador:

Se realizó la calcinación de los óxidos en una mufla a 550oC durante cuatro horas para incrementar su pureza (Albis et al 2005). En orden de evitar la inactivación de los centros activos se procedió a su protección mediante su mezcla a $1200 \mathrm{RPM}$ con metanol a 50oC en condiciones de reflujo por 30 minutos de acuerdo a las proporciones estequeométricas establecidas en cada tratamiento. 


\section{Producción de biodiesel a escala de laboratorio:}

La transesterificación se llevó a cabo en un reactor discontinuo conformado por un balón de tres vías de $500 \mathrm{ml}$ conectado a un condensador de reflujo. La temperatura se monitoreó con un termómetro digital acoplado a una de las vías laterales del recipiente. La tercera vía sirvió para alimentar los reactivos al equipo.

El sistema de calefacción se conformó por una placa calefactora con sistema de agitación magnético. Se dispuso un recipiente de vidrio sobre la placa, dentro del cual reposó el reactor para garantizar el uniforme calentamiento del balón por medio de baño maría.

La reacción tomó lugar al añadir la grasa de pollo pre tratada al equipo por medio de un embudo. Una vez cerrado el reactor, se abrió el suministro de agua de refrigeración para el condensador y se fijó la temperatura a $60 \mathrm{oC}$. Se controló la duración del proceso mediante un cronómetro.

\section{Purificación de subproductos:}

La purificación del biodiesel constó de tres etapas: destilación, decantación y filtración. Se acopló el balón empleado como reactor para destilar el metanol en exceso. Posteriormente, se trasvasó el contenido no destilado a un embudo de decantación donde reposó por 24 horas para permitir la separación de los productos de la transesterificación en diferentes fases. Luego de este período, se observó una fase pesada correspondiente a la glicerina, una intermedia con catalizador y una ligera de biodiesel. Las fases obtenidas se filtraron individualmente al vacío.

\section{Caracterización del biodiesel obtenido:}

Los ensayos efectuados para la caracterización del biodiesel obtenido bajo las mejores condiciones de reacción fueron: punto de inflamación, sedimentos básicos y agua, contenido de azufre, índice de cetano, corrosión a la lámina de cobre, viscosidad cinemática, densidad a 15oC y ensayo de destilación. Los resultados de estos parámetros fueron contrastados con los rangos establecidos en la norma INEN 1489 para determinar la calidad del biocombustible.

\section{Tratamiento estadístico de los resultados:}

El análisis estadístico contempló la determinación de los intervalos de confianza para el rendimiento de la reacción respecto a cada variable de estudio para determinar las mejores condiciones experimentales. Los análisis fueron efectuados utilizando el programa estadístico Statgraphics Centurion presentando un nivel de confianza del 95,0\%. Complementariamente, se realizó un análisis de la varianza ANOVA para determinar si las diferencias en el rendimiento de la reacción causadas por cada factor fueron estadísticamente significativas tomando como criterio todos los valores-P menores a 0,05 .

\section{Resultados}

\section{Caracterización de la Grasa Residual recuperada del proceso de cocción:}


El perfil lipídico de la grasa reveló que priman con 54,20\% ácidos grasos mono insaturados, principalmente el ácido oléico con una concentración del 45,55\%. Los ácidos grasos saturados constituyen el 31,94\% de la muestra, siendo el ácido palmítico el preponderante de esta clasificación con un 24,95\%. En menor proporción se encuentran los ácidos grasos poli insaturados con $13,68 \%$. Se obtuvo un índice de acidez de apenas $0,1 \%$ lo que indica niveles mínimos de ácidos grasos libres.

El índice de saponificación permite estimar de manera indirecta el contenido de triglicéridos de una muestra. El valor promedio obtenido (309,74 $\mathrm{mg} \mathrm{KOH} / \mathrm{g}$ de grasa) es alto por lo cual la materia prima empleada es idónea para la realización del proceso de transesterificación. Se debe puntualizar que este valor también es un referente de la producción de jabón que pudiese generarse si las condiciones de reacción no fuesen adecuadas...

Los ensayos determinaron que el índice de peróxido promedio fue de 99,33 miliequivalentes de oxígeno por kilogramo de muestra, este valor se encuentra veinte veces por arriba del límite superior establecido para el rango de aceites y grasas vírgenes el cual va de 0-5 miliequivalentes de oxígeno por kilogramo de muestra. En base a este valor se puede esperar una baja estabilidad oxidativa en el biodiesel resultante.

\section{Tratamientos con Oxido de Calcio:}

En la totalidad de experimentos planteados con este catalizador se generó la transesterificación. El rendimiento máximo obtenido fue de 90,23\% y el mínimo de 13,41\%.

La figura 1 evidencia claramente mayor efectividad con una relación molar alcohol-aceite de 12:1 mostrando en todos los casos rendimientos sobre el 50\%. El uso del 1\% en peso de catalizador generó rendimientos mayores respecto al valor superior asignado a esta variable.

El contenido de jabón como subproducto no deseado fue detectado en todos los ensayos. El valor máximo registrado fue de 29,99 g y el mínimo de 4,69 g correspondientes a los tratamientos T9 y T13 respectivamente (Figura1). Se debe acotar que los experimentos descritos coinciden con aquellos que presentaron el menor y mayor rendimiento de biodiesel lo cual es congruente puesto que lo que no se transesterifica tiende a saponificarse.

En relación a los otros subproductos, se obtuvo un máximo de glicerina de $38,0 \mathrm{~mL}$ y un mínimo de 14,00 ml. La tasa promedio de recuperación de metanol fue de 55,15\% siendo la del tratamiento de mayor rendimiento de 59,34\%. La medición del catalizador gastado se realizó por diferencia de peso tras la filtración. Se obtuvo en el ensayo T13 un valor de 0,80 g de catalizador en base húmeda representando el $50 \%$ de la cantidad dosificada en el proceso. 


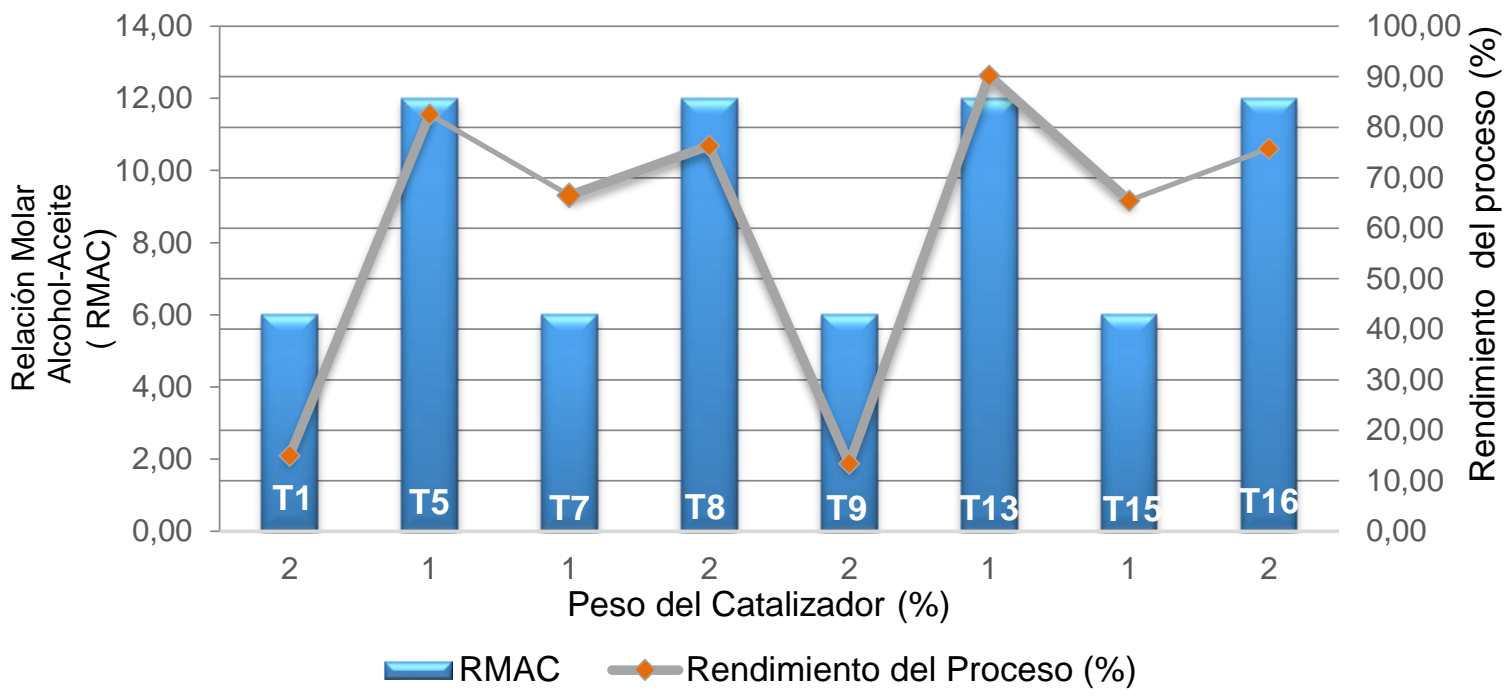

Figura 1: Rendimiento de los tratamientos con $\mathrm{CaO}$.

\section{Tratamientos con Oxido de Magnesio:}

Los experimentos con óxido de magnesio demostraron que a pesar de producir biodiesel en todos los ensayos la efectividad del proceso tiende a ser baja. El mayor rendimiento registrado fue de $22,17 \%$ y el menor de 2,37\% correspondientes a los tratamientos T10 y T3 respectivamente (Figura 2).

La figura 2 indica que todos los procesos llevados a cabo con una relación molar alcoholaceite $6: 1$ obtuvieron rendimientos menores al 5\%. Igualmente se aprecia que los ensayos que emplearon un $1 \%$ en peso de catalizador indistintamente de la RMAC obtuvieron mejores resultados con relación aquellos realizados con un $2 \%$ cuyos rendimientos no sobrepasaron el $11,34 \%$.

La grasa sin reaccionar causada por la baja conversión del proceso fue el principal subproducto no deseado. Su volumen máximo fue de $138 \mathrm{ml}$ y el mínimo de $80 \mathrm{ml}$. En cuanto a los otros subproductos, se registró una tasa de recuperación de metanol del 45,90\%. La glicerina obtenida estaba dentro del rango de 1,20 a 17,00 ml. Los valores sobre el catalizador gastado en base húmeda presentaron un máximo de 21,66 g y un mínimo de 6,28g. 


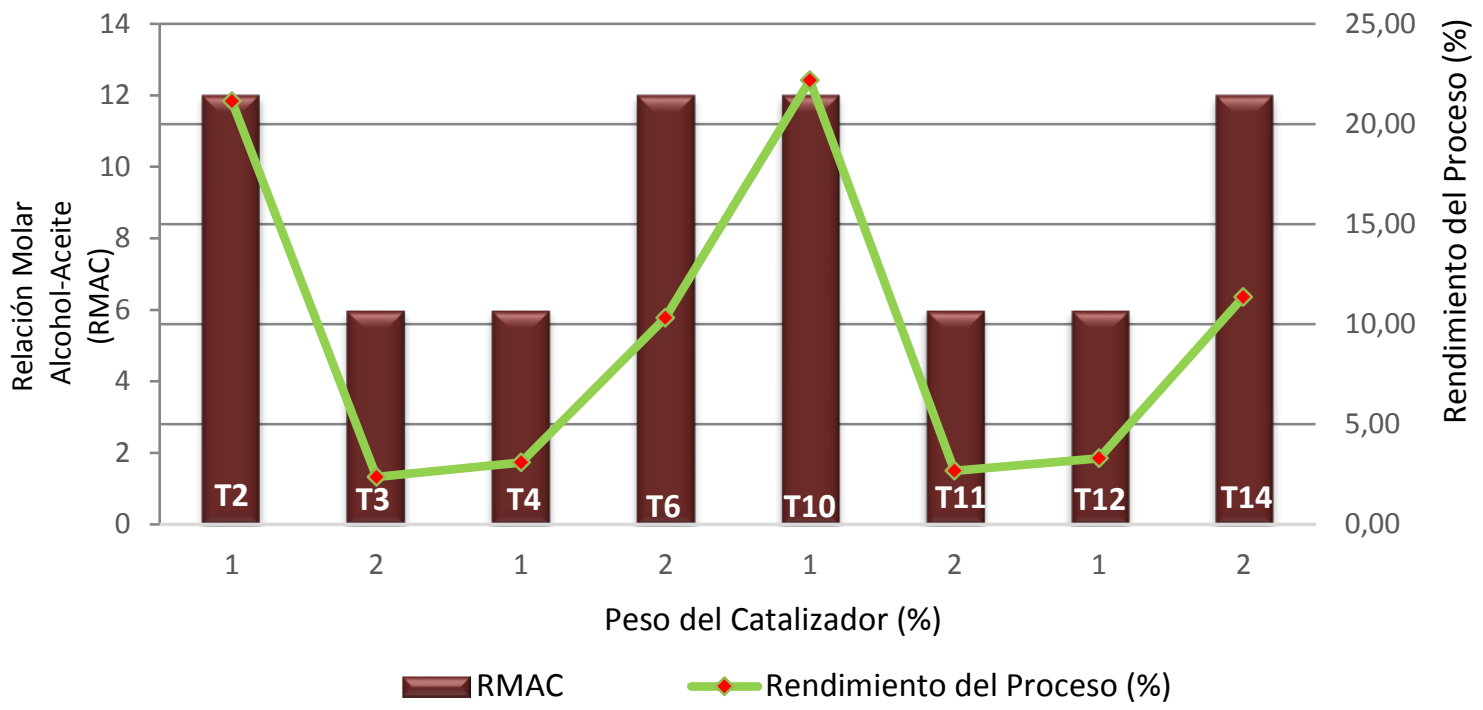

Figura 2: Rendimiento de los tratamientos con $\mathrm{MgO}$.

\section{Análisis Estadístico:}

Con base en los límites de confianza detallados en la tabla 3 se evidencia que las mejores condiciones de transesterificación con ambos catalizadores fueron una relación molar alcoholaceite de $12: 1$ y un $1 \%$ de catalizador.

En el caso de los tratamientos con óxido de calcio, los límites inferiores calculados para el rendimiento de la reacción en cada uno de los niveles óptimos sobrepasan el 58\% y los superiores son mayores al $96 \%$. Por otro lado, los ensayos realizados con óxido de magnesio reflejaron una baja efectividad puesto que ninguno de sus límites superiores sobrepasa el 20,41\% de rendimiento.

Tabla 3: Límites de confianza de los tratamientos

\begin{tabular}{ccccc}
\hline $\begin{array}{c}\text { Tipo de } \\
\text { Catalizador }\end{array}$ & Factor & Nivel & \multicolumn{2}{c}{ Rendimiento de la Reacción (\%) } \\
\cline { 3 - 4 } & & & & \\
\cline { 3 - 4 } & & 6 & $\begin{array}{c}\text { Límite } \\
\text { Inferior }\end{array}$ & Limite Superior \\
& RMAC & 22,91 & 57,27 \\
& & 12 & 64,03 & 98,39 \\
& PPC & 2 & 59,00 & 96,36 \\
& & 6 & 0,00 & 62,3 \\
& RMAC & 12 & 12,07 & 7,03 \\
& & 1 & 8,25 & 20,41 \\
MgO & PPC & 2 & 2,50 & 16,60 \\
& & & & 10,85 \\
\hline
\end{tabular}


La tabla 4 detalla los valores $\mathrm{P}$ obtenidos del análisis ANOVA. A partir de los resultados se evidencia que el porcentaje en peso de óxido de calcio y su relación molar alcohol-aceite son variables significativas y por tanto inciden directamente en el rendimiento de la reacción. Por otro lado, el porcentaje en peso de óxido de calcio presentó un valor mayor a 0,05 ; lo cual indica que las diferencias estadísticas debido a sus distintos niveles no fueron significativas y por tanto el rendimiento no depende directamente de este factor

Tabla 4: Análisis ANOVA en base a los tratamientos

\begin{tabular}{lll}
\hline Catalizador & Efectos Principales & Valor P \\
\hline $\mathbf{C a O}$ & RMAC & 0,0074 \\
& PPC & 0,0218 \\
& & \\
$\mathbf{M g O}$ & RMAC & 0,0021 \\
& PPC & 0,0542 \\
\hline
\end{tabular}

\section{Caracterización del biodiesel resultante:}

La tabla 5 indica los parámetros evaluados en el biodiesel obtenido bajo las mejores condiciones de operación, las cuales fueron una RMAC 12:1 y un 1\% de óxido de calcio como catalizador.

Tabla 5: Caracterización del biodiesel obtenido

\begin{tabular}{|c|c|c|c|c|c|c|}
\hline \multirow{3}{*}{ Parámetros } & \multirow{3}{*}{ Unidad } & \multirow{3}{*}{$\begin{array}{c}\text { Valor } \\
\text { Obtenido }\end{array}$} & \multirow{2}{*}{\multicolumn{2}{|c|}{$\begin{array}{c}\text { Requisitos Biodiesel } \\
\text { Norma INEN } 2482\end{array}$}} & \multirow{2}{*}{\multicolumn{2}{|c|}{$\begin{array}{cc}\text { Requisitos } & \text { Diesel No.2 } \\
\text { Norma INEN } 1489\end{array}$}} \\
\hline & & & & & & \\
\hline & & & Mínimo & Máximo & Mínimo & Máximo \\
\hline Densidad a $15^{\circ} \mathrm{C}$ & $\mathrm{Kg} / \mathrm{m}^{3}$ & 825 & 860 & 890 & - & - \\
\hline Punto de Inflamación & ${ }^{\circ} \mathrm{C}$ & 63 & 120 & - & 51 & - \\
\hline $\begin{array}{l}\text { Corrosión lámina de } \\
\text { cobre }\end{array}$ & - & $1 \mathrm{~A}$ & - & 3 & - & 3 \\
\hline $\begin{array}{l}\text { Temperatura de } \\
\text { destilación al } \\
\text { 90\%recuperado }\end{array}$ & ${ }^{\circ} \mathrm{C}$ & 337 & - & 360 & - & 360 \\
\hline $\begin{array}{c}\text { Viscosidad Cinemática } \\
\mathrm{a} 40^{\circ} \mathrm{C}\end{array}$ & $\mathrm{mm}^{2} / \mathrm{s}$ & 3,848 & 3,5 & 5 & 2,0 & 5,0 \\
\hline Índice de Cetano & - & 48,3 & 49 & - & 45 & - \\
\hline Agua y sedimentos & $\%$ & $<0,05$ & - & 0,05 & - & 0,05 \\
\hline Contenido de azufre & $\%$ & 0,023 & - & - & - & 0,7 \\
\hline
\end{tabular}

\section{Producción estimada de los supermercados:}


Una vez completada la fase experimental se procedió a realizar una estimación de la capacidad productiva de biodiesel de las veinte sucursales que cuenta la cadena de supermercados, ubicadas en Quito.

El cálculo se realizó en base a los datos obtenidos y a la producción estimada de grasa residual del supermercado tomado como base para esta investigación. Se estableció como rendimiento del proceso un $82,50 \%$ correspondiente al valor promedio obtenido bajo las mejores condiciones de reacción. La tabla 6 resume la producción esperada tanto mensual como anualmente.

Tabla 6: Capacidad Productiva Supermercados

\begin{tabular}{lll}
\hline Capacidad Productiva & \multicolumn{1}{c}{ (20 locales) } & Unidades \\
\hline $\begin{array}{l}\text { Producción de Grasa de Pollo Residual } \\
\text { Producción de Grasa de Pollo Residual }\end{array}$ & 1260,00 & $\mathrm{~L} / \mathrm{mes}$ \\
Producción de Biodiesel & 1094,94 & $\mathrm{Kg} / \mathrm{mes}$ \\
Producción de Glicerina & 1503,33 & $\mathrm{Kg} / \mathrm{mes}$ \\
Producción de Biodiesel & 1094,94 & $\mathrm{Kg} / \mathrm{mes}$ \\
Producción de Glicerina & 126,18 & L/mes \\
Producción Anual de Grasa de Pollo Residual & 15120 & L/mes \\
Producción de Biodiesel & 13139,28 & L/año \\
Producción de Glicerina & 1514,15 & L/año \\
\end{tabular}

\section{Conclusiones}

\section{Caracterización de la Grasa de Pollo Residual:}

En cuanto al índice de acidez, el valor obtenido de $0,1 \%$ se encuentra debajo del rango establecido ( $\geq 3 \%$ ) para implementar la esterificación como pretratamiento; razón por la cual la única medida necesaria para el acondicionamiento de esta materia prima es un proceso de filtración al vacío.

Las propiedades del biodiesel dependen directamente de la concentración y el tipo de ácidos grasos presentes en la materia prima. A mayor contenido de ácidos grasos saturados incluyendo al mono insaturado disminuye la viscosidad e incrementa el número de cetano del biodiesel. En su conjunto los ácidos grasos mencionados constituyeron el 86,14\% de la grasa de pollo residual, razón por la cual el cumplimiento de los parámetros indicados resultó congruente.

\section{Rendimiento de los Tratamientos:}


De acuerdo a los intervalos de confianza al 95\%, las mejores condiciones para la transesterificación empleando óxido de calcio como catalizador son una relación molar alcoholaceite de 12:1 y 1\% de catalizador. El límite de confianza superior para el rendimiento del proceso correspondiente a una relación molar 12:1 (98,39\%) sobrepasa en 71,80\% al límite superior determinado para la proporción 6:1. En el caso del porcentaje en peso, el límite superior para esta condición establece un rendimiento superior al $98 \%$.

En el caso del óxido de magnesio los análisis estadísticos reflejaron rendimientos bajos mostrando intervalos de confianza con límites superiores que no sobrepasaron el $20,41 \%$ de rendimiento.

El análisis ANOVA, el valor P de 0,0542 reveló que el porcentaje en peso de óxido de magnesio no es una variable significativa por lo que no incide directamente en el rendimiento del proceso. Ante este criterio se concluye que el uso de este catalizador no es efectivo y debe descartarse como variable de estudio.

\section{Caracterización del Biodiesel:}

De acuerdo a la norma INEN 2482 referente a los requisitos del biodiesel se determinó que el biocombustible resultante del tratamiento con óxido de calcio bajo las mejores condiciones de reacción cumple los siguientes requisitos: corrosión a la lámina de cobre, viscosidad cinemática, contenido de azufre, temperatura de destilación al $90 \%$ recuperado y contenido tanto de agua como de sedimentos.

Una de las ventajas presentadas por el biocombustible obtenido fue un contenido de azufre del 0,023\% el cual es 30 veces menor al límite permisible para el diésel No.2. Esta reducción significativa se reflejará en menor producción de emisiones de dióxido de azufre en la combustión minimizando impactos ambientales y mejorando la calidad del aire ambiente.

Si se considera los requisitos específicos para el diésel No.2, el cual es utilizado en automotores a diésel y en equipos industriales, los parámetros de estudio cumplieron en su totalidad los requisitos por lo que el biocombustible podría utilizarse para los equipos descritos bien sea puro o como mezcla.

La capacidad productiva conjunta determinada para los establecimientos en Quito fue de: 15120 L/año de grasa de pollo residual, la cual bajo las mejores condiciones de operaciones determinadas experimentalmente para la transesterificación produciría 13139,28 L/año de biodiesel y 1514,15L/año de glicerina. Estos valores referenciales pueden ser un aporte importante para evaluar a través de un estudio de pre factibilidad la posibilidad de adoptar este sistema productivo enmarcado en los principios de la producción más limpia.

\section{Bibliografía}

Alba, A. (2011). Nuevos procesos para la obtención de biodiesel a partir de catalizadores ácidos y básicos. Tesis de doctorado no publicada. Universidad Autónoma de Madrid, Madrid, España. 
Albis, A. Parra, J. Sánchez, F. (2005). Transesterificación del aceite de palma con metanol por catálisis heterogénea. Ingeniería e Investigación, vol.25, pp.71-77

Becera, M. López, J Centeno, A. Giraldo, A. (2008). Producción de Biodiesel y Glicerina Limpia Empleando Catálisis Básica Heterogénea. Revista ION, vol. 21, pp.31-38

Castellar, G. Angulo, E. Cardoso, B. (2014). Transesterificación de aceites vegetales empleando catalizadores heterogéneos. Prospect, vol.12, pp.90-104.

Dinis. (2012). Obtención de biodiesel por transesterificación de aceites vegetales: nuevos métodos de síntesis. Tesis de doctorado no publicada. Universidad de Extremadura, Extremadura, España.

Galeano, C. Guapacha, E. (2011). Aprovechamiento y caracterización de los residuos grasos de pollo para la producción de biocombustibles (biodiesel). Tesis de grado sin publicar. Universidad

Tecnológica de Pereira, Pereira, Colombia.

Guayara, A. Rodríguez, A. Rubio, J. Gallegos, W. (2016). Estudio de pre-factibilidad del proceso de obtención de biodiesel a partir de la grasa de pollo recuperada de los residuos generados en su proceso de cocción. Tesis de grado no publicada. Universidad Internacional Sek- Ecuador. Quito, Ecuador.

Kawashima, A. Matsubara, K. Honda, K. (2009). Acceleration of catalytic activity of calcium oxide for biodiesel production. Bioresource Technology, vol. 100, pp.696-700

MacLeod, C. S., Harvey, A. P., Lee, A. F., Wilson, K. (2008). Evaluation of the activity and stability of alkali-doped metal oxide catalysts for application to an intensified method of biodiesel production. Chemical Engineering Journal, vol. 135, pp. 63-70.

Medina, E. Acosta, A. Burgos, A. Crisanto, B. Eyzaguirre, P. Rivera, J. (2013). Análisis y diseño de un sistema de recolección y tratamiento de aceites domésticos usados para la producción de biodiesel en la ciudad de Piura y Castilla. Universidad de Piura.

Montenegro, M., Sierra, F., Guerrero, C. (2012). Producción y caracterización de biodiésel a partir de aceite de pollo. Informador Técnico, vol. 76, pp.62-71

Ramírez. Chávez, N. Jauregui, J. (2012). Biodiesel, un combustible renovable. Investigación y Ciencia de la Universidad Autónoma de Aguascalientes, vol. 55, pp.62-70.

Rojas, A. Girón, E. Torres, H. (2009). Variables de operación en el proceso de transesterificación de aceites vegetales: una revisión-catálisis química. Ingeniería e Investigación, vol.29, pp.17-22 
Salamatinia, B., Hashemizadeh, I., Ahmad Zuhairi, A. (2013). Alkaline Earth Metal Oxide Catalysts for Biodiesel Production from Palm Oil: Elucidation of Process Behaviors and Modeling Using Response Surface Methodology. Iranian Journal of Chemistry and Chemical Engineering (IJCCE), vol 32, pp. 113-126

Tejada, C., Tejeda, L., Villabona, A., Monroy, L. (2013). Obtención de biodiesel a partir de diferentes tipos de grasa residual de origen animal. Revista luna azul, número 36, pp. 1025. 